BNL-113215-2016-CP

File \#94164

\title{
Simulation of an IXS imaging analyzer with an extended scattering source
}

\author{
Alexey Suvarov and Yong Q. Cai \\ Presented: SPIE Conference Proceedings \\ Conference title: SPIE Optics and Photonics Conference \\ San Diego, CA
}

Aug 28 - Sep 1, 2016

Physics Department

Brookhaven National Laboratory

\author{
U.S. Department of Energy \\ USDOE Office of Science (SC), \\ Basic Energy Sciences (BES) (SC-22)
}

Notice: This manuscript has been authored by employees of Brookhaven Science Associates, LLC under Contract No. DE- SC0012704 with the U.S. Department of Energy. The publisher by accepting the manuscript for publication acknowledges that the United States Government retains a non-exclusive, paid-up, irrevocable, world-wide license to publish or reproduce the published form of this manuscript, or allow others to do so, for United States Government purposes. 


\section{DISCLAIMER}

This report was prepared as an account of work sponsored by an agency of the United States Government. Neither the United States Government nor any agency thereof, nor any of their employees, nor any of their contractors, subcontractors, or their employees, makes any warranty, express or implied, or assumes any legal liability or responsibility for the accuracy, completeness, or any third party's use or the results of such use of any information, apparatus, product, or process disclosed, or represents that its use would not infringe privately owned rights. Reference herein to any specific commercial product, process, or service by trade name, trademark, manufacturer, or otherwise, does not necessarily constitute or imply its endorsement, recommendation, or favoring by the United States Government or any agency thereof or its contractors or subcontractors. The views and opinions of authors expressed herein do not necessarily state or reflect those of the United States Government or any agency thereof. 


\title{
SPIE, ADVANCES IN X-RAY/EUV OPTICS AND COMPONENTS XI CONFERENCE
}

San Diego, CA; Aug 28 - Sep 1, 2016

BNL-113215-2016; File \# 94164

\section{Simulation of an IXS imaging analyzer with an extended scattering source}

\author{
Alexey Suvorov*a, Yong Q. Cai ${ }^{\mathrm{a}}$ \\ ${ }^{a}$ National Synchrotron Light Source II, Brookhaven National Laboratory, Upton, NY 11973, USA
}

\begin{abstract}
A concept of an inelastic x-ray scattering (IXS) spectrograph with an imaging analyzer was proposed recently and discussed in a number of publications (see e.g. Ref.1). The imaging analyzer as proposed combines x-ray lenses with highly dispersive crystal optics. It allows conversion of the x-ray energy spectrum into a spatial image with very high energy resolution. However, the presented theoretical analysis of the spectrograph did not take into account details of the scattered radiation source, i.e. sample, and its impact on the spectrograph performance. Using numerical simulations we investigated the influence of the finite sample thickness, the scattering angle and the incident energy detuning on the analyzer image and the ultimate resolution.
\end{abstract}

Keywords: Inelastic x-ray scattering, $\mathrm{x}$-ray spectrograph, $\mathrm{x}$-ray optics, numerical simulation

\section{INTRODUCTION}

Conceptually, a spectrograph is an instrument that separates the incoming electro-magnetic radiation into a frequency spectrum and records the signal using a camera. It allows one to obtain a snapshot of the whole spectrum as an image on a spatially sensitive detector. Contrary to a conventional spectrum analyzer which processes every spectrum point sequentially, the acquisition time of a spectrograph can be drastically reduced. Therefore, it is highly desirable to have a spectrograph with a high energy resolution for inelastic x-ray scattering (IXS) studies. Implementation of an IXS spectrograph in the hard $\mathrm{x}$-ray regime requires the combination of focusing and energy dispersing crystal optics. A proof-of-principle prototype of such a hard x-ray spectrograph with a resolving power of $E / \Delta E \gtrsim 10^{8}$ (at $E \simeq 9.1 \mathrm{keV}$ ) was first demonstrated by Y.Shvyd' $\mathrm{ko}^{2,3}$. To achieve the high resolving power, the monochromator of the spectrograph must have an energy bandwidth of the same order or better. Taking into account weak IXS signal, practical implementation of the IXS spectrograph would require an x-ray source with greatly enhanced spectral flux.

To overcome limitations in spectral resolution and weak signals of the IXS technique, an X-ray echo spectroscopy was recently introduced ${ }^{1}$. The echo spectrograph performs point-to-point focusing of a polychromatic x-ray beam using a defocusing monochromator and a refocusing analyzer. Analysis of the echo spectrograph indicated its theoretical feasibility of achieving the energy resolution of $\Delta E \cong 0.1 \mathrm{meV}$ in the hard x-ray range. Utilization of the broadband monochromator and analyzer provides a high gain in the signal strength compared to the other types of IXS spectrometers.

The presented analysis of the echo spectrograph ${ }^{1}$ was based on the geometrical optics approximation and used the raytransfer matrix technique to propagate x-rays through the system. It assumes that a hypothetical sample is located in the infinitely thin plane in-between the defocusing monochromator and the refocusing analyzer. However, factors like the finite geometrical dimensions of a sample, the scattering angle and monochromator energy detuning can potentially impact the imaging properties of the echo spectrograph and therefore worsen its energy resolution. In the present paper, we analyze the influence of these factors on the performance of the spectrograph using numerical simulations based on the wave-optical approach to the wave propagation through the system.

*asuvorov@bnl.gov; phone 1631 344-7096; fax 1631 344-8189 


\section{Spectrograph outline}

\section{ANALYSIS AND SIMULATIONS}

The specific design of the echo spectrograph can be found in Ref.1. Its schematic outline is shown in Fig.1. The crystal optics parameters of the spectrograph at photon energy $E \simeq 9.1 \mathrm{keV}$ are summarized in Table 1 . The focal distances of the lenses $L 1, L 2$ and $L 3$ are $f_{1}=2.0 \mathrm{~m}, f_{2}=0.4 \mathrm{~m}$ and $f_{3}=0.4 \mathrm{~m}$ respectively. The sample plane is located at the rear focal point of the lens $L 1$ and at the front focal point of the lens $L 2$. It was assumed that the distances between the crystals and from the crystals to the lenses are negligible.

Table 1. Basic crystal parameters of the defocusing monochromator and the refocusing analyzer in the simulated echo spectrograph. The last column shows the absolute values of the asymmetry factors.

\begin{tabular}{|c|c|c|c|c|c|}
\hline \multicolumn{6}{|c|}{ Monochromator } \\
\hline Crystal & Material & $\begin{array}{l}\text { Reflection } \\
(h k l)\end{array}$ & $\begin{array}{l}\text { Bragg angle } \\
\text { (deg) }\end{array}$ & $\begin{array}{l}\text { Asymmetry } \\
\text { angle (deg) }\end{array}$ & $\begin{array}{l}\text { Asymmetry } \\
\text { factor }\end{array}$ \\
\hline M1 & $\mathrm{Ge}$ & $\left(\begin{array}{lll}1 & 1 & 1\end{array}\right)$ & 12.0 & -10.0 & 0.09 \\
\hline M2 & $\mathrm{Si}$ & $\left(\begin{array}{lll}8 & 0 & 0\end{array}\right)$ & 89.0 & 77.5 & 1.17 \\
\hline M3 & $\mathrm{Si}$ & $\left(\begin{array}{lll}8 & 0 & 0\end{array}\right)$ & 89.0 & 77.5 & 1.17 \\
\hline M4 & $\mathrm{Ge}$ & $\left(\begin{array}{lll}1 & 1 & 1\end{array}\right)$ & 12.0 & 10.0 & 10.8 \\
\hline \multicolumn{6}{|c|}{ Analyzer } \\
\hline Crystal & Material & $\begin{array}{l}\text { Reflection } \\
(h k l)\end{array}$ & $\begin{array}{l}\text { Bragg angle } \\
\text { (deg) }\end{array}$ & $\begin{array}{l}\text { Asymmetry } \\
\text { angle (deg) }\end{array}$ & $\begin{array}{l}\text { Asymmetry } \\
\text { factor }\end{array}$ \\
\hline A1 & $\mathrm{Ge}$ & $\left(\begin{array}{lll}1 & 1 & 1\end{array}\right)$ & 12.0 & -10.0 & 0.09 \\
\hline A2 & $\mathrm{Si}$ & $\left(\begin{array}{lll}8 & 0 & 0\end{array}\right)$ & 89.0 & -86.0 & 0.6 \\
\hline A3 & $\mathrm{Si}$ & $\left(\begin{array}{lll}8 & 0 & 0\end{array}\right)$ & 89.0 & -86.0 & 0.6 \\
\hline A4 & $\mathrm{Ge}$ & $\left(\begin{array}{lll}1 & 1 & 1\end{array}\right)$ & 12.0 & 10.0 & 10.8 \\
\hline
\end{tabular}

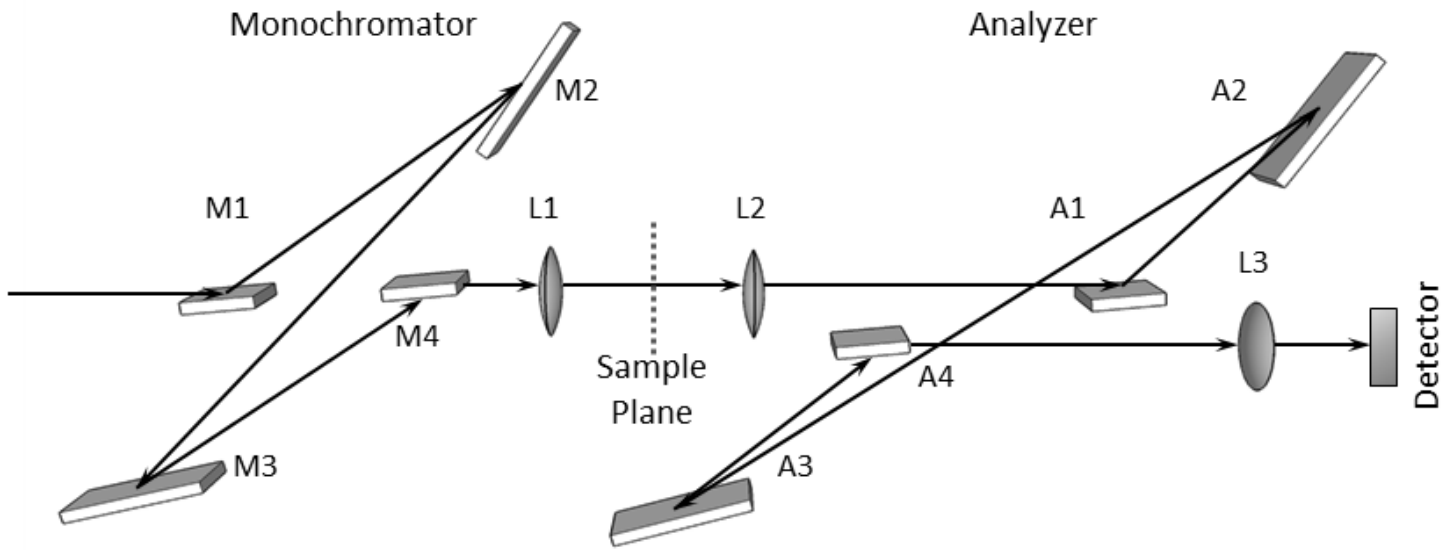

Figure 1. Schematic outline of the simulated echo spectrograph comprising the defocusing monochromator and the refocusing analyzer separated from each other by the sample plane.

A quasi-monochromatic beam transmitted through the defocusing monochromator exhibits an angular dispersion in the diffraction plane of the crystal optics. The monochromator dispersion changes the angle of propagation of the monochromatic components of the beam .With the crystal parameters shown in Table 1, the cumulative dispersion rate 
of the monochromator is $D_{M}=-25 \mu \mathrm{rad} / \mathrm{meV}$. Following the monochromator the lens $L 1$ performs spatial separation of the monochromatic components of the beam in the sample plane $e^{4,5}$. The dimensions of the x-ray source and its distance to the monochromator were set to achieve a $5 \mu \mathrm{m} \times 5 \mu \mathrm{m}(\mathrm{VxH})$ focal spot in the center of the sample plane at the central energy $E_{0}=9.133 \mathrm{keV}$. Small deviation of the x-ray energy from the central one moves the monochromatic focal spot in the vertical plane of the sample. By this means, the defocusing monochromator produces a quasi-monochromatic beam elongated in the vertical direction of the sample plane. The vertical spread of the beam can be estimated as $\Delta_{V}=D_{M} f_{1} \Delta E_{M}$ where $\Delta E_{M}$ is the energy bandwidth of the monochromator. The calculated energy bandwidth of the monochromator was $\Delta E_{M}=9.4 \mathrm{meV}$ and the corresponding spatial spread of the beam was about $470 \mu \mathrm{m}$. Because of this vertical stretch of the quasi-monochromatic beam at the sample, the monochromator was called defocusing.

The refocusing analyzer can be considered as a mirror or echo image of the monochromator. It transmits the beam from the sample through the crystal optics and focuses it into a single spot in the detector plane. It can be interpreted in a way that the reverse propagation of a quasi-monochromatic beam from a source in the detector plane should produce the same pattern in the sample plane as the defocusing monochromator. The lens $L 2$ is located at the focal length $f_{2}$ from the sample and is acting as a beam collimator for the crystal optics. The lens L3 images the transmitted beam onto the position sensitive detector located in the rear focal plane. However, the proposed design of the refocusing analyzer is not an exact replica of the defocusing monochromator. The difference in the focal distances of the monochromator lens $L 1$ and the analyzer lens $L 2$ results in a narrower energy bandwidth accepted by the analyzer than that of the monochromator. While the monochromator provides a beam with the energy bandwidth of $9.4 \mathrm{meV}$, the accepted beam by the analyzer has a bandwidth of only about $2 \mathrm{meV}$. The energy acceptance of the analyzer is limited due to the angular acceptance of the $A 1$ crystal. Although the crystals $M 4$ and $A 1$ are similar, the focal distances $f_{1}$ and $f_{2}$ differs by a factor of 5 which leads to the reduction of the energy acceptance by the same factor. Another distinction of the analyzer is the cumulative asymmetry factor $b_{A}=0.36$ and dispersion rate $D_{A}=-43.5 \mu \mathrm{rad} / \mathrm{meV}$. The cumulative asymmetry factor changes the magnification factor of the analyzer resulting in the vertical focal spot size of about $2 \mu \mathrm{m}$ versus $5 \mu \mathrm{m}$ in the sample plane. The higher dispersion rate of the analyzer provides larger spatial deviation of the focused beam with the energy variation. Because of this the ultimate energy resolution of the analyzer attains the desirable $0.1 \mathrm{meV}$.

\section{Finite sample thickness}

In the general analysis of the echo spectrograph ${ }^{1}$ the sample was replaced by an infinitely thin sample plane. In real experiments samples have finite dimensions. Sample thickness in the IXS experiments may depend on the sample state, specific enclosure, x-ray absorption length etc., and may vary widely from a fraction of a millimeter to several

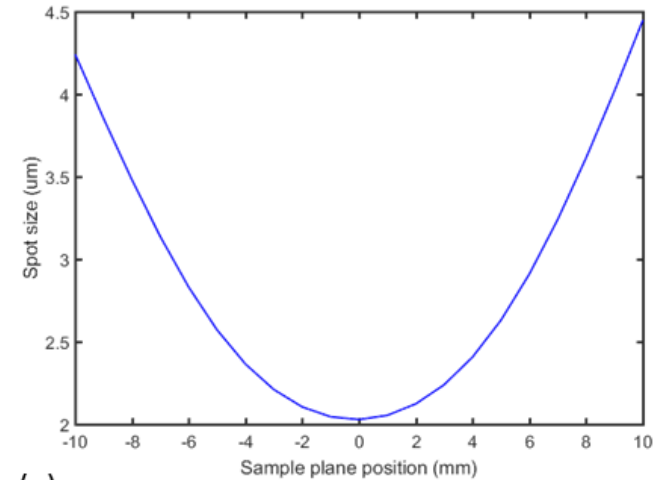

(a)

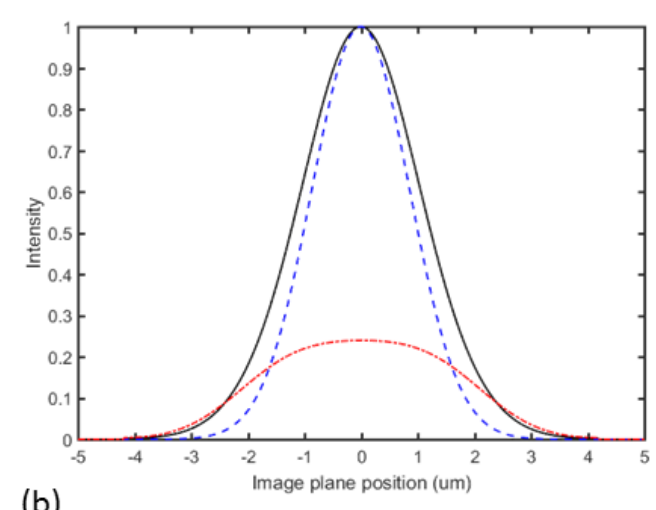

(b)

Figure 2. Vertical dimension of the refocusing analyzer focal spot for different locations of the sample plane (a) and the focal spot intensity profiles (b). Negative sample plane position in (a) corresponds to the shift of the sample plane upstream from the nominal position. The blue and red intensity profiles in (b) corresponds to the sample plane displacement of 0 and $-10 \mathrm{~mm}$ respectively. The black profile in (b) is the normalized intensity integrated over the sample plane positions.

millimeters. With the finite thickness of the sample the secondary source of the scattered radiation is extended at different distances from the lens $L 2$. This can potentially lead to broadening of the focal spot in the detector plane and consequently to the worsening of the energy resolution of the refocusing analyzer. We have performed numerical simulations of the analyzer 2D images for the various locations of the secondary source in the forward scattering 
geometry. It was assumed that the detector has a finite spatial resolution of $0.5 \mu \mathrm{m}$. Figure 2(a) demonstrates the focal spot broadening versus the displacement of the secondary scattering plane from the nominal position. It can be seen that the vertical dimension of the focused beam gets doubled at the displacement of about $9 \mathrm{~mm}$ from the central plane. Figure 2(b) compares intensity profiles in the vertical plane of the detector. The blue curve corresponds to the zero position of the secondary source plane and represents the best-focused beam with the spot size of about $2 \mu \mathrm{m}$. The red curve corresponds to the $10 \mathrm{~mm}$ shift upstream from the zero position and represents the defocused beam with the spot size of about $4 \mu \mathrm{m}$. As it can be expected, the defocused beam profile gets broader and lower in peak intensity. The black curve in the Fig.2(b) shows the normalized intensity integrated over all scattering planes within the range of \pm 10 $\mathrm{mm}$. Although at the extreme front and back locations of the scattering source the analyzer focal spot is largely defocused, the integration over the plane position diminishes the broadening and the integrated intensity has the focal spot size of about $2.5 \mu \mathrm{m}$ and leads to an energy resolution degradation by $25 \%$.

\section{Non-zero scattering angle}

The combination of the finite sample thickness with a non-zero scattering angle results in a horizontal broadening of the secondary source of the scattered radiation (Fig.3(a)). The effects are twofold. First, the analyzer image gets broader.
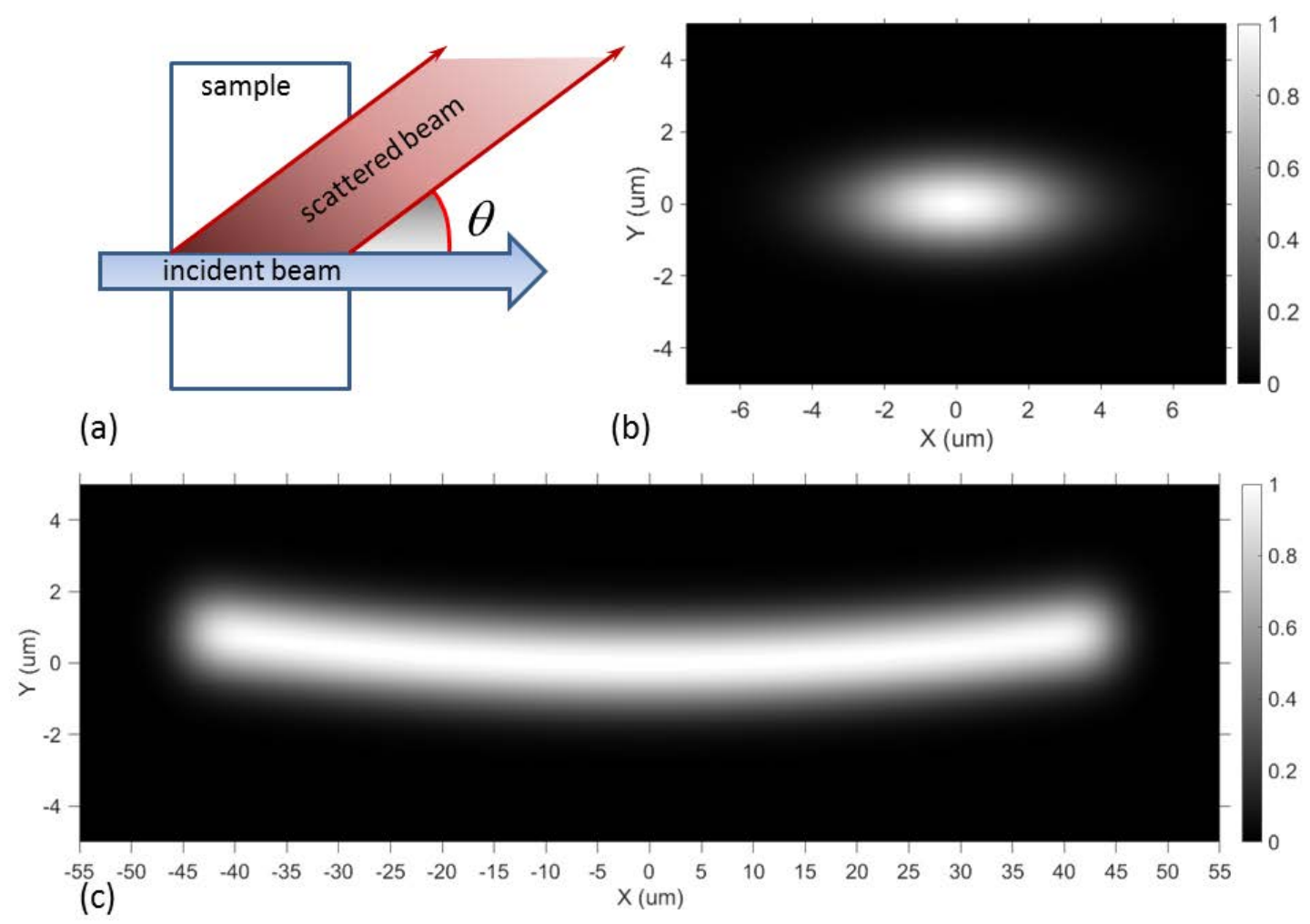

Figure 3. Top view of the scattering geometry (a) and the refocusing analyzer images at scattering vectors (b) $Q=0 \mathrm{~nm}^{-1}$ ( $\theta=0 \mathrm{deg}$ ), and (c) $Q=4 \mathrm{~nm}^{-1}$ ( $\theta=5 \mathrm{deg}$ ). The intensity in the images is normalized. The hypothetical sample thickness was $1 \mathrm{~mm}$.

While the integrated intensity is conserved, the intensity per unit area (per detector pixel) of the image plane is diminished. The horizontal dimension of the analyzer image at $\theta=0^{\circ}$ is about $5 \mu \mathrm{m}$. The analyzer image at $\theta=5^{\circ}$ is broadened by a factor of about 18 . Because of this the intensity reduction in the analyzer image can diminish or outweigh the gain from the enhanced energy bandwidth of the incoming beam.

Secondly, the image gets a banana shape due to the propagation of the beam out of the diffraction/dispersion plane. Broadening of the secondary source of scattered x-rays in the horizontal plane introduces an azimuthal angle of incidence to the crystal optics. As a result, the wave vector projection onto the diffraction/dispersion plane gets reduced 
and leads to the displacement of the beam in the vertical plane. Although this is a second order effect, it needs to be taken into account if one wants to integrate the intensity over the horizontal axis of the detector.

\section{Monochromator energy detuning}

With the given parameters of the refocusing analyzer (Table 1), its energy bandwidth is about $5.3 \mathrm{meV}$. To image an inelastic signal with a larger energy transfer, the defocusing monochromator energy has to be detuned. This requires detuning of the angles of incidence of the monochromator crystals. There are four crystals in the monochromator design. While the crystals $M 1$ and $M 4$ have a low reflection order and wide energy bandwidth, the crystals $M 2$ and $M 3$ have a high reflection order and narrow energy bandwidth. The detuning of only the crystals $M 2$ and $M 3$ can change the energy of the monochromatic beam within the range of about $25 \mathrm{meV}$ which is limited by the energy bandwidths of these crystals. The only way to detune the energy of the defocusing monochromator by more than $25 \mathrm{meV}$ is to change the angular positions of all four crystals. In the linear approximation the magnitude of the detuning angle can be derived from the Bragg's law

$$
\Delta \theta=-\frac{\Delta E_{M}}{E_{0}} \tan \theta_{B}
$$

where $\Delta E_{M}=E_{M}-E_{0}, E_{M}$ and $E_{0}$ are the monochromator detuned and nominal energies respectively, and $\theta_{B}$ is the Bragg angle of the reflection.

The detuning of the energy of the defocusing monochromator also changes its cumulative dispersion rate. Figure 4 illustrates the dependence of the cumulative dispersion rate of the monochromator on the energy of the transmitted beam. It has a very close to linear variation in the energy range of about $\pm 250 \mathrm{meV}$. The variation of the cumulative

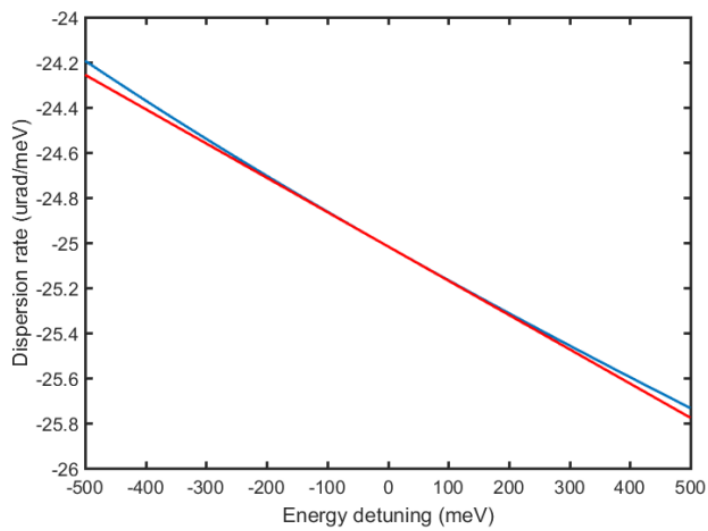

Figure 4. Variation of the dispersion rate of the defocusing monochromator with the energy of the transmitted beam. The blue line shows the simulated data and the red line shows a linear analytical approximation.

dispersion rate occurs due to the change of the asymmetry factors of the crystals. In accordance with Eq.1, crystals $M 2$ and $M 3$ require larger angular deviations per same energy detuning than the other two crystals. Also they have much larger asymmetry angles than the other two crystals. Because of this, these two crystals are the main contributors to the dispersion rate variation with energy. Taking into account the identical parameters of crystals $M 2$ and $M 3$, the simplified analytical expression for the dispersion rate variation around the nominal value is

$$
\Delta D_{M} \approx-4 b_{4}\left(1+b_{2}\right) \frac{\sin ^{2} \varphi_{2} \tan \theta_{2}}{\sin ^{2}\left(\theta_{2}-\varphi_{2}\right)} \frac{\Delta E_{M}}{E_{0}^{2}}
$$

where $b_{n}$ is the asymmetry factor of the n-th crystal, and $\varphi_{2}$ and $\theta_{2}$ are the asymmetry angle and the Bragg angle of the second crystal respectively. Eq. 2 is plotted in the Fig. 4 as a red line and provides a very good approximation in a wide energy rage.

Suppose that the monochromator energy was detuned in such a way that the inelastic energy transfer by the sample matches the detuning. Then upstream from the sample the beam central energy is $E_{\mathrm{M}}$, and downstream from the sample the beam central energy is $E_{0}$. The change in the dispersion rate due to the detuning results in a different distribution of the monochromatic components in the sample plane. After the sample, the ultimate deflection of the monochromatic focal spot in the detector plane due to the dispersion rate variation can be expressed as 


$$
\Delta y=b_{A} \Delta D_{M} \Delta E f_{1}
$$

where $\Delta E=E-E_{0}$ is the deviation from the beam central energy and $f_{1}$ is the focal length of the lens $L 1$. For example, the energy detuning of the defocusing monochromator by $250 \mathrm{meV}$ leads to the deviation of the dispersion rate from the nominal value by about $\Delta D_{M}=-0.4 \mu \mathrm{rad} / \mathrm{meV}$. As we mentioned earlier, the energy acceptance of the refocusing analyzer is about $\pm 1 \mathrm{meV}$. Assuming $\Delta E=1 \mathrm{meV}$, Eq.3 gives the deflection of the focused beam in the detector plane by about $\Delta y=-0.3 \mu \mathrm{m}$. This is a very small deflection compare to the nominal focal spot size of $2 \mu \mathrm{m}$. In addition, integration of the intensity over the accepted energy bandwidth of the analyzer produces a beam spot virtually indistinguishable from that without the detuning of the monochromator energy.

\section{CONCLUSIONS}

The newly proposed echo spectrograph was tested numerically under conditions close to a real IXS experiment. The simulated scattering from a hypothetical sample with a finite thickness and a non-zero scattering angle demonstrated the ability of the spectrograph to maintain its high energy resolution in a wide range of the varied parameters. To a large extent, this was ensured by the long focal distances, $f_{2}$ and $f_{3}$, and small cumulative asymmetry factor, $b_{\mathrm{A}}$, of the refocusing analyzer. The long focal distances make the analyzer insensitive to the variation of scattering plane distance within several millimeters. The small cumulative asymmetry factor make the analyzer insensitive to the dispersion rate variation due to the monochromator energy detuning. One of the potential advantages of the proposed spectrograph is the opportunity to make the compact analyzer design. However, this can only be done at the expense of the solid angle acceptance of the analyzer. For example, with the grazing angle of $3^{\circ}$ the $200 \mathrm{~mm}$ long crystals $A 2$ and $A 3$ can accept a $\Delta_{2}=10 \mathrm{~mm}$ incident beam. Then, the dimension of the accepted beam upstream from the crystal $A 1$ is reduced to $\Delta_{1}=b_{\mathrm{A} 1} \Delta_{2}=1 \mathrm{~mm}$. With the focal length $f_{2}=0.4 \mathrm{~m}$ of the collimating lens $L 2$ the angular acceptance of the analyzer is limited to $\Delta_{1} / f_{2}=2.5 \mathrm{mrad}$. This number is far smaller than that of the other types of the IXS spectrometers. Another inconvenient feature of the spectrograph is the diminution of the image intensity with the secondary source expansion. While the integrated intensity of the analyzer image is conserved, the intensity per unit area of the position sensitive detector depends on the dimensions of the secondary source in the sample plane. Depending on the sample thickness and scattering angle, the transverse dimension of the secondary source can be significantly extended. As a result, the intensity in the detector plane can be diminished by an order or more of the magnitude.

\section{ACKNOWLEDGMENTS}

This research used resources of the National Synchrotron Light Source II, a U.S. Department of Energy (DOE) Office of Science User Facility operated for the DOE Office of Science by Brookhaven National Laboratory under Contract No. DE-SC0012704.

\section{REFERENCES}

[1] Shvyd'ko, Y., “X-ray echo spectroscopy,” Phys. Rev. Lett. 116, 080801 (2016).

[2] Shvyd'ko, Y., "Enhanced x-ray angular dispersion and x-ray spectrographs with resolving power beyond 108," Proc. SPIE 8502, 85020J (2012).

[3] Shvyd'ko, Y., Stoupin, S., Mundboth, K. and Kim, J., "Hard-x-ray spectrographs with resolution beyond $100 \mu \mathrm{eV}$," Phys. Rev. A 87(4), 043835.(2013).

[4] Shvyd'ko, Y., “Theory of angular-dispersive, imaging hard-x-ray spectrographs,” Phys. Rev. A 91(5), 053817 (2015).

[5] Suvorov, A., Cunsolo, A., Chubar, O., and Cai, Y. Q., "Ultrahigh energy resolution focusing monochromator for inelastic x-ray scattering spectrometer,” Optics express, 23(24), 31607-31618 (2015). 